\title{
Ivabradine Versus Beta-Blockers in Patients with Conduction Abnormalities or Left Ventricular Dysfunction Undergoing Cardiac Surgery
}

Luminita Iliuta $\cdot$ Marius Rac-Albu

To view enhanced content go to www.cardiologytherapy-open.com

Received: October 2, 2013 / Published online: December 17, 2013

(c) The Author(s) 2013. This article is published with open access at Springerlink.com

\section{ABSTRACT}

Introduction: In patients with conduction abnormalities or left ventricle (LV) dysfunction the use of $\beta$-blockers for post cardiac surgery rhythm control is difficult and controversial, with a paucity of information about other drugs such ivabradine used postoperatively. The objective of this study was to compare the efficacy and safety of ivabradine versus metoprolol used perioperatively in cardiac surgery patients with conduction abnormalities or LV systolic dysfunction.

Methods: This was an open-label, randomized clinical trial enrolling 527 patients with

Electronic supplementary material The online version of this article (doi:10.1007/s40119-013-0024-1) contains supplementary material, which is available to authorized users.

L. Iliuta · M. Rac-Albu ( $\varangle)$

"Carol Davila" University of Medicine and

Pharmacy, Bucharest, Romania

e-mail: racalbu@yahoo.com

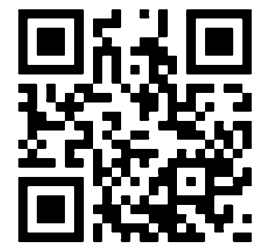

Enhanced content for this article is

available on the journal web site:

www.cardiologytherapy-open.com conduction abnormalities or LV systolic dysfunction undergoing coronary artery bypass grafting or valvular replacement, randomized to take ivabradine or metoprolol, or metoprolol plus ivabradine. The primary endpoints were the composites of 30-day mortality, in-hospital atrial fibrillation (AF), in-hospital three-degree atrioventricular block and need for pacing, inhospital worsening heart failure (HF; safety endpoints), duration of hospital stay and immobilization and the above endpoint plus in-hospital bradycardia, gastrointestinal symptoms, sleep disturbances, cold extremities (efficacy plus safety endpoint).

Results: Heart rate reduction and prevention of postoperative AF or tachyarrhythmia with combined therapy was more effective than with metoprolol or ivabradine alone during the immediate postoperative management of cardiac surgery patients. In the Ivabradine group, the frequency of early postoperative pacing and HF worsening was smaller than in the Metoprolol group and in combined therapy group. The frequency of primary combined endpoint was lower in the combined Ivabradine + Metoprolol group compared with the monotherapy groups. 
Conclusion: Considering efficacy and safety, the cardiac rhythm reduction after open heart surgery in patients with conduction abnormalities or LV dysfunction with ivabradine plus metoprolol emerged as the best treatment in this trial.

Keywords: Atrial fibrillation; Beta-blocker; Cardiac surgery; Cardiology; Coronary artery bypass surgery; Ivabradine; Metoprolol; Postoperative arrhythmias; Valvular replacement

\section{INTRODUCTION}

Postoperative arrhythmias are serious and common complications following open heart surgery and are associated with increased morbidity and mortality [1]. Older age is the most consistent predictor of postoperative atrial arrhythmias [1]. Atrial fibrillation (AF) is the most common arrhythmia encountered postoperatively [1], with a reported incidence from 20 to 40\%, although ventricular arrhythmias and conduction disturbances can also occur [2]. Patients who develop postoperative arrhythmias are more likely to have other postoperative complications such as perioperative myocardial infarction, congestive heart failure (HF), respiratory failure and increased hospital length of stay and costs [39]. Recent studies report incidences of atrial arrhythmias after coronary artery bypass grafting (CABG) surgery to be $17-33 \%$ [3-8], and even higher after valvular surgery [4].

While postoperative atrial fibrillation (POAF) can be transient and without consequences, it may lead to serious complications such as increased risk of acute kidney injury, hemodynamic instability, cardiac failure, stroke, and death [2]. In spite of older age being still the most consistent predictor of postoperative $\mathrm{AF}$, factors such as acute atrial changes occurring at the time of surgery (acute atrial enlargement, intraoperative atrial ischemia), hypertension, trauma from cannulation, hypomagnesaemia, inflammation caused by pericarditis, cardiopulmonary bypass and cross-clamp times, pulmonary vein venting and also excessive adrenergic stimulation, are incriminated in its occurrence in vulnerable patients [9-15].

Postoperative atrial fibrillation is most often detected on the second and third day postoperatively [12-15] and is frequently selflimiting and short-lived. Up to $80 \%$ of patients convert to sinus rhythm (SR) within $24 \mathrm{~h}$, and 6 weeks after initial diagnosis $98 \%$ of patients have converted to SR [5].

Patients developing postoperative AF are more likely to have prolonged hospitalization and intensive care unit (ICU) stay and therefore, increased economic burden of their care $[7,8]$. Despite the identification of risk factors and efforts to determine effective prophylactic agents, postoperative atrial arrhythmias remain a prominent clinical issue.

The meta-analyses and systematic reviews for prevention of postoperative rhythm disorders showed that interventions to prevent and/or treat postoperative AF with $\beta$-blockers, sotalol or amiodarone and, less convincingly, override suppression of automatic atrial foci by atrial pacing, are favored with respect to outcome (AF occurrence, stroke, and length of hospitalization) [16-21]. Despite multiple randomized studies demonstrating the consistent prophylactic effectiveness of $\beta$-blockers [16-21], in most trials, $\beta$-blockers have been used only in a limited manner, presumably because of fear of hemodynamic or pulmonary intolerance and excluding patients with significant obstructive lung disease, atrioventricular (AV) block greater than first degree and impaired left ventricle (LV) function $[9,22]$. 
Because the use of $\beta$-blockers is difficult and controversial in patients with conduction abnormalities, severe LV dysfunction, active bronchospasm, there is a need for another drug for rhythm control in postoperative cardiac surgical patients.

The selective $I_{\mathrm{f}}$ current inhibitor ivabradine reduces heart rate without affecting cardiac contractility by selective sinus node inhibition, and has been shown to be cardio protective in the failing heart [23].

Ivabradine showed improvement of clinical outcomes in patients with stable coronary artery disease and LV systolic dysfunction [24] or chronic HF [23]. However, there are few published data from clinical trials which evaluated the efficacy and benefits of Ivabradine used postoperatively in patients with conduction abnormalities or LV dysfunction undergoing coronary surgery for prevention or treatment of postoperative rhythm disorders [25].

Ivabradine also exerts some of its beneficial effects by decreasing cardiac proinflammatory cytokines and inhibiting peroxidants and collagen accumulation in atherosclerosis or congestive heart failure [26].

The main objectives of our study were to compare the efficacy and safety of heart rate lowering agent ivabradine versus $\beta$-blocker metoprolol used perioperatively in patients undergoing cardiac surgery and having conduction abnormalities (first degree AV block or bundle branch block) or LV dysfunction and also to determine whether prophylactic therapy with ivabradine can reduce hospital stay and economic costs after cardiac surgery by lowering the risk associated with an increased heart rate.

\section{METHODS}

This clinical trial was open-label, randomized, enrolling 527 patients undergoing cardiac surgery (CABG with arteries-internal mammary, radial, gastroepiploic-or inverted saphenous veins, valvular replacement or combined interventions) in a single center (Cardiac Surgery Department of Emergency Institute for Cardiovascular Diseases "Prof. Dr. C. C. Iliescu", Bucharest, Romania) between January 1, 2006 and December 31, 2010. Surgical management and treatment of the patients were based on a common standard protocol.

The study was not registered with any international body prior to commencement.

\section{Eligibility Criteria}

Patients included in the clinical trial were patients undergoing elective cardiac surgery interventions who had conduction abnormalities, LV systolic dysfunction or both. At the moment of the enrolment into the study all patients were in sinusal rhythm.

\section{Exclusion Criteria}

Not eligible for the study were the patients exhibiting one or more of the following conditions: third degree AV block; bradycardia [heart rate less than 50 beats per minute (bpm)] or conditions associated with increased risk for bradycardia (vagal predominance, sick sinus syndrome); HF New York Heart Association (NYHA) class IV; cardiogenic shock; severe chronic obstructive pulmonary disease or pulmonary impairment; known hypersensitivity to $\beta$-blockers or ivabradine; active participation in another clinical trial; failure to comply with the hospital protocol or absence to follow-up.

Study drop-out criteria included the occurrence of adverse events: severe bradycardia, skin reactions, gastrointestinal 
symptoms, cold extremities. The study protocol was approved by the institute Management and Ethics Committee. All procedures followed were in accordance with the ethical standards of the responsible committee on human experimentation (institutional and national) and with the Helsinki Declaration of 1975, as revised in 2000 and 2008. Informed consent was obtained from all patients for being included in the study.

\section{Study Groups}

After inclusion in the study, 2 days before surgery, patients were randomized in three groups: Metoprolol group: 176 patients to receive metoprolol $100 \mathrm{mg}$ orally once daily; Metoprolol + Ivabradine group: 179 patients to receive metoprolol $50 \mathrm{mg}$ orally once daily and ivabradine $5 \mathrm{mg}$ orally twice daily; Ivabradine group: 172 patients to receive ivabradine $5 \mathrm{mg}$ orally twice daily. During the postoperative nil by mouth period ivabradine was administered via nasogastric tube. The treatment phase comprised 2 days preoperatively and at least 10 days postoperatively and the patients were followed up for 30 days after surgery (Fig. 1). The study drug was withdrawn in patients who developed postoperative AF as there is no effect of ivabradine on heart rate in this situation.

\section{Clinical and Laboratory Assessments}

Patients were evaluated at baseline (i.e., 2 days before surgery), daily from Day 1 until Day 10 postoperatively, on Day 15, and at the end of the treatment on Day 30 postoperatively. Patients with short in-hospital evolution were evaluated ambulatory.

Clinical parameters included NHYA class, ventricular rhythm, patient compliance, and

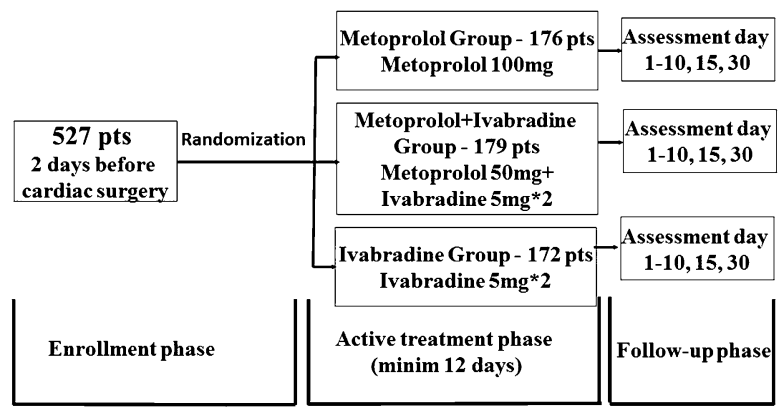

Fig. 1 Study phases and distribution of study population. Minim at least, $p t s$ patients

quality of life (QOL). QOL was measured by the EuroQol questionnaire [27].

Laboratory parameters assessed were: usual blood tests (white and red blood cell count, platelet count, hemoglobin, hematocrit, alanine aminotransferase and aspartate aminotransferase, lactate dehydrogenase, blood chemistry), electrocardiogram (ECG) (with the evaluation of rhythm and rate), $24 \mathrm{~h}$ ECG Holter monitoring and echocardiographic measurements of the LV dimensions, LV systolic and diastolic performance, left atrium dimensions and compliance (data not shown in the present report). Cardiac rhythm was continuously monitored in the intensive care unit. During further hospital stay, subsequent ECG tests and a $24 \mathrm{~h}$ ECG Holter monitoring was carried out prior to discharge.

Follow-up visits were in Day 15 and in Day 30 postoperatively and included a physical examination and a 15-min interview, a resting ECG, an echocardiogram and a $24 \mathrm{~h}$ ECG Holter monitoring. Early episodes of HF were diagnosed based on clinical signs and symptoms and by transthoracic and transesophageal echocardiography. The presence of bradycardia or second or third degree AV block was assessed using clinical examination, resting ECG and $24 \mathrm{~h}$ ECG Holter monitoring. 


\section{Study Endpoints}

The efficacy endpoints were 30-day mortality, in-hospital occurrence of AF/arrhythmias, inhospital occurrence of third degree AV block and need for pacing, in-hospital worsening heart failure and duration of hospitalization and immobilization. Safety endpoints were occurrence of bradycardia, gastrointestinal complaints, sleep disturbances, and cold extremities. A composite efficacy and safety endpoint including 30-day mortality, inhospital AF/arrhythmias, in-hospital AV block/ need for pacing, or in-hospital heart failure worsening was also defined.

\section{Statistical Analyses}

No sample size assumptions have been made for this trial. Continuous variable are presented as mean \pm standard deviation. Categorical variables are displayed as percentages. To analyze the differences between the treatment groups, the Student's $t$ test was used for the continuous variables and the Chi square test for the categorical variables. For each endpoint, a two-sided 95\% confidence interval was calculated and an overall Chi square test comparing the two treatment groups was used. Also, we performed simple and multivariate, linear and logistic regression analysis and we calculated relative risks and correlation coefficients. For the primary endpoints Kaplan-Meier curves were constructed and log-rank tests were used. All statistical analyses were performed using SYSTAT 12 (Systat Software, Inc., IL, USA) and SPSS Statistics 18 (IBM Corporation, NY, USA) software. A $P$ value less than 0.05 defined the statistical significance.

\section{RESULTS}

In the entire study population, mean age was $63 \pm 8$ years, and $40.99 \%$ of patients were female. Baseline demographics and clinical characteristics of the three treatment groups are displayed in Table 1.

There were no differences in age and gender of patients, presence of LV dysfunction or conduction abnormalities between study groups, systolic blood pressure or mean baseline heart rate, mean number of grafts/ patient and grafts type, type of valvular replacement, risk score for atrial arrhythmias and mean treatment duration. The percentages of patients with previous episodes of $\mathrm{AF}$, with LV dysfunction and conduction abnormalities (first degree AV block, complete left bundle branch block, bifascicular and trifascicular block) were similar in the three groups.

Study groups structure depending on the type of the surgical intervention and on the type of the relative contraindication to $\beta$ blockers is presented in Fig. 2 .

The primary efficacy and safety, single and composite endpoints in the treatment groups are shown in Table 2.

In-hospital postoperative AF or tachyarrhythmias occurred less frequently with combined therapy (metoprolol and ivabradine) than with metoprolol or ivabradine alone $(P<0.001)$. The associated relative risk showed a higher protective value for the occurrence of postoperative AF in patients undergoing cardiac surgery interventions treated with combined therapy compared with metoprolol monotherapy ( -2.9 versus -1.8 ; Fig. 3 ).

In the Ivabradine group, the frequency of early postoperative third degree AV block or need for pacing and also the frequency of HF worsening was lower than in the Metoprolol group and in the combined Metoprolol + Ivabradine group 
Table 1 Baseline demographics and clinical characteristics of study population by treatment group

\begin{tabular}{llll}
\hline Characteristics $^{\mathbf{a}}$ & $\begin{array}{l}\text { Metoprolol } \\
\text { group } \\
\mathbf{N = 1 7 6}\end{array}$ & $\begin{array}{l}\text { Metoprolol + Ivabradine } \\
\text { group } \\
\mathbf{N}=\mathbf{1 7 9}\end{array}$ & $\begin{array}{l}\text { Ivabradine } \\
\text { group } \\
\mathbf{N}=\mathbf{1 7 2}\end{array}$ \\
\hline Age (years) & $63(12)$ & $63(12)$ & $63(13)$ \\
Percentage female & $39.77 \%$ & $40.78 \%$ & $42.44 \%$ \\
Weight (kg) & $76(16)$ & $75(14)$ & $77(13)$ \\
Height (cm) & $171(9)$ & $173(10)$ & $170(8)$ \\
Mean heart rate (over 24 h) & $81(15)$ & $80(16)$ & $79(14)$ \\
LV systolic dysfunction & $42.61 \%$ & $43.02 \%$ & $44.19 \%$ \\
Conduction abnormalities & $39.77 \%$ & $39.66 \%$ & $38.37 \%$ \\
LV dysfunction and conduction abnormalities & $17.61 \%$ & $17.32 \%$ & $17.44 \%$ \\
Previous episodes of atrial arrhythmias & $20.45 \%$ & $20.11 \%$ & $20.35 \%$ \\
Hypertension & $65.34 \%$ & $65.92 \%$ & $68.60 \%$ \\
Diabetes mellitus & $28.98 \%$ & $29.61 \%$ & $27.33 \%$ \\
Re-intervention-previous CABG & $9.66 \%$ & $10.05 \%$ & $9.30 \%$ \\
Re-intervention-previous valve replacement & $2.27 \%$ & $2.23 \%$ & $3.49 \%$ \\
CABG & $59.66 \%$ & $60.33 \%$ & $59.30 \%$ \\
Valve replacement & $32.39 \%$ & $32.40 \%$ & $33.72 \%$ \\
CABG and valve replacement & $7.95 \%$ & $7.26 \%$ & $6.98 \%$ \\
\hline
\end{tabular}

$C A B G$ coronary artery bypass grafting, $L V$ left ventricle

${ }^{a}$ Parameters are expressed as mean values (standard deviation) or percentages. All $P$ values for comparisons between groups were not significant

$(P<0.0001 ;$ Table 2$)$. Also, the associated relative risks for early postoperative complete AV block or need for permanent pacing and for postoperative HF worsening were lower in ivabradine-treated groups (Fig. 3).

The rates of combined efficacy plus safety endpoints (30-day mortality, in-hospital AF/ arrhythmias, in-hospital AV block/need for pacing, or in-hospital HF worsening) were lower in the combined therapy group versus metoprolol or ivabradine monotherapy groups $(P<0.0001$; Table 2$)$.

The overall QOL was better in the Ivabradine group. Ivabradine-treated patients had shortened hospital stay (the mean duration of hospital stay in the Metoprolol group was $10.2 \pm 6.3$ days, compared to $8.5 \pm 6.8$ days in the Metoprolol + Ivabradine group and $8.2 \pm 6.4$ days in the Ivabradine group), and reduced immobilization duration in the immediate postoperative period $(2.0 \pm 3.0$ days in the Metoprolol group, $1.1 \pm 3.0$ days in the Metoprolol + Ivabradine group and $1.1 \pm 3.0$ days in the Ivabradine group). Also, the percent of the patients with hospitalization duration $>15$ days or with immobilization period $>3$ days was smaller in the combined Metoprolol + Ivabradine group $\quad(P<0.005$; Table 2). The cumulative incidence of noncardiac side effects (sleep disturbances, 


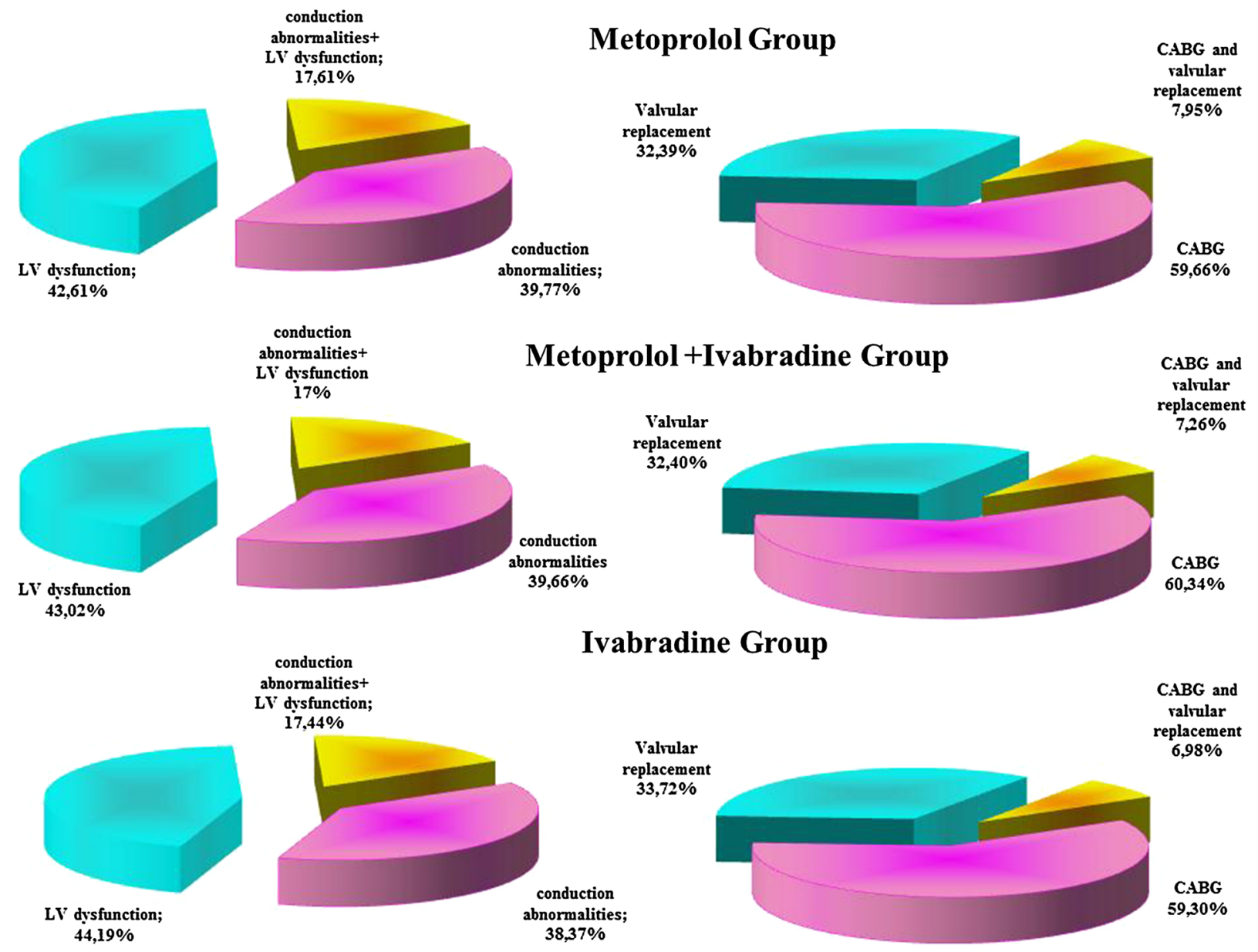

Fig. 2 Study groups structure. $C A B G$ coronary artery bypass grafting. $L V$ left ventricle

gastrointestinal symptoms, and skin reactions) was similar in the study groups (Table 2).

The superiority of ivabradine (efficacy and safety) was also shown by Kaplan-Meier curves generated for the primary endpoints in both groups (ivabradine as monotherapy and combination therapy ivabradine plus metoprolol), log-rank tests being highly significant between days 4-30 of treatment (Fig. 4).

In Fig. 3 we indicate associated relative risks in ivabradine-treated groups (with or without metoprolol) comparative with Metoprolol group after cardiac surgery according to type of surgical intervention, preoperative conduction abnormalities, previous episodes of
AF, NYHA class, grafts number, and age. It is obvious the superiority of ivabradine (as monotherapy or combined therapy with metoprolol) versus metoprolol therapy alone in terms of efficacy and safety endpoint of 30-day mortality, in-hospital HF worsening, inhospital AF/arrhythmias or in-hospital AV block/need for pacing.

\section{DISCUSSION}

The present study is one of the first studies that evaluated the efficacy and safety of ivabradine therapy for prevention of postoperative AF or other tachyarrhythmias in patients undergoing coronary or valvular surgery. Prevention of 
Table 2 Composite and single efficacy and safety endpoints by treatment group

\begin{tabular}{|c|c|c|c|}
\hline Endpoints & $\begin{array}{l}\text { Metoprolol } \\
\text { group } \\
N=176(\%)\end{array}$ & $\begin{array}{l}\text { Metoprolol + Ivabradine } \\
\text { group } \\
N=179(\%)\end{array}$ & $\begin{array}{l}\text { Ivabradine } \\
\text { group } \\
N=172(\%)\end{array}$ \\
\hline 30-day mortality, in-hospital AF/arrhythmias & 13.64 & 12.85 & 23.84 \\
\hline $\begin{array}{l}\text { 30-day mortality, in-hospital AF/arrhythmias, in-hospital } \\
\text { AV block/need for pacing, or in-hospital HF worsening }\end{array}$ & 34.09 & 23.46 & 29.07 \\
\hline Death at 30 days & 3.98 & 3.91 & 4.07 \\
\hline In-hospital AF/arrhythmias & 9.66 & 8.94 & 19.77 \\
\hline In-hospital 3 degree AV block/need for pacing & 12.50 & 6.15 & 2.91 \\
\hline In-hospital HF worsening & 7.95 & 4.47 & 2.33 \\
\hline Hospitalization duration $>15$ days & 33.52 & 17.88 & 23.26 \\
\hline Immobilization for $>3$ days & 25.00 & 16.76 & 22.67 \\
\hline Sleep disturbances/gastrointestinal symptoms/skin reactions & 3.41 & 3.35 & 3.49 \\
\hline
\end{tabular}

$A F$ atrial fibrillation, $A V$ atrioventricular, $H F$ heart failure

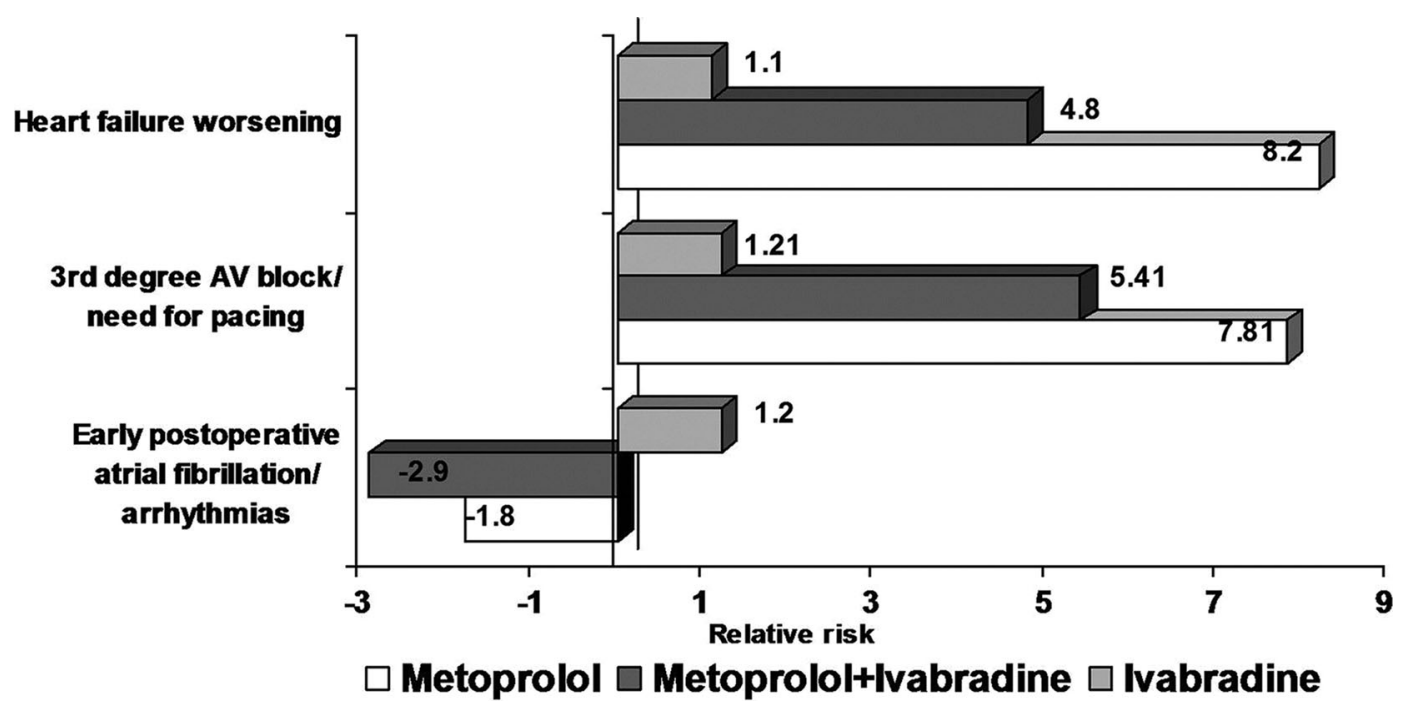

Fig. 3 The relative risks of ivabradine and combined therapy with ivabradine and metoprolol versus metoprolol monotherapy for early postoperative atrial fibrillation,

postoperative rhythm disorders immediately after cardiac surgery becomes increasingly important because they are associated with longer ICU and hospital stays (up to 5 days) [28, 29], significantly higher (two to threefold) risk of postoperative stroke [28-31], increased morbidity and mortality, with consecutively complete atrioventricular block/need for pacing and postoperative heart failure worsening. $A V$ atrioventricular

important economic burden of these outcomes. Although the overall topic of AF after cardiac surgery was evaluated in a lot of clinical trials, still, there is a relative paucity of evidence-based studies dealing with pharmacologic heart rhythm control addressing this topic [31]. 


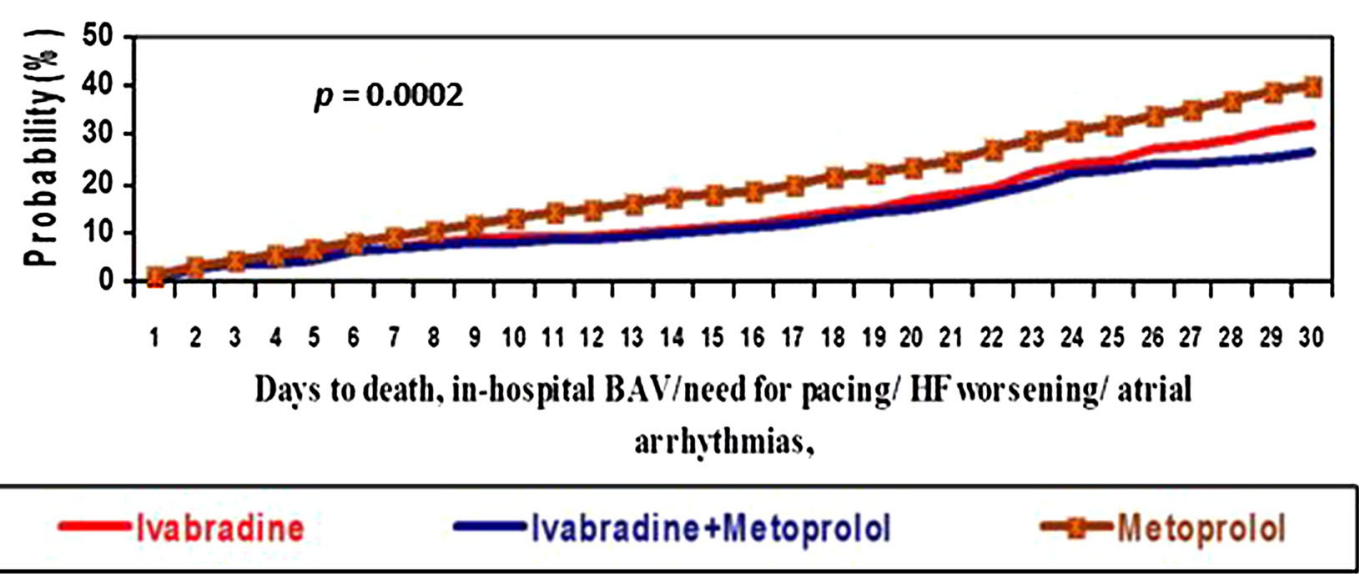

Fig. 4 Kaplan-Meier curves for the composite endpoint of 30-day mortality, in-hospital atrial fibrillation/arrhythmias, in-hospital atrioventricular block/need for pacing, or inhospital heart failure worsening in the three treatment

Atrial fibrillation is the most common complication which occurs after cardiac surgery, with frequencies ranging from 30\% after coronary artery bypass grafting, $40 \%$ after valve surgery, and $50 \%$ after combined coronary artery bypass grafting/valve surgery [9]. Development of AF immediately after coronary artery bypass grafting or after valve surgery or combined requires a longer period of hospitalization [28, 29], an increased risk (two to threefold) of postoperative stroke [28, 30] and also it independently predict postoperative delirium and neurocognitive decline [17]. The risk categories for POAF include those with prolonged cross-clamp time, chronic obstructive pulmonary disease, advanced age, atrial ischemia, proximal right coronary artery disease, and withdrawal of beta-blockers (should be avoided before surgery being a significant risk factor for POAF) [9].

At present, $\beta$-blockers are the mainstay of therapy for prevention of postoperative $\mathrm{AF}$ in cardiac surgery being recommended both by the American College of Cardiology/American Heart Association (ACC/AHA) 2004 Guideline update for $C A B G$ surgery and by the most recent groups: ivabradine alone versus combined ivabradine plus metoprolol and metoprolol alone. $B A V$ atrioventricular block, $H F$ heart failure

European Society of Cardiology (ESC) guidelines for the management of AF as a class I indication in the prophylactic management of POAF in patients without contraindications to $\beta$-blocker therapy $[9,22]$.

Studies showed that withdrawal of betablockers in the perioperative period doubles the incidence of PAOF after coronary artery bypass grafting [22]. Virtually every study of beta-blockers administered for the purpose of reducing POAF has shown benefit in this regard, even if data regarding improvement of hospital stay or reduction of stroke incidence are still controversial [16]. Most beta-blockers trials have examined the initiation of prophylaxis in the postoperative period. But it seems to be an even greater benefit if beta-blocker therapy is initiated before surgery. That is why the ESC guidelines for the management of $\mathrm{AF}$ recommend that treatment should be started at least 1 week before surgery with a beta1blocker without intrinsic sympathomimetic activity [9].

The $\beta$-blockers used in studies assessing AF prevention in cardiac surgery were propranolol [19], atenolol [32], metoprolol [18, 21], 
carvedilol [33, 34], acebutolol [35], timolol [36], betaxolol [16], compared to control or to another $\beta$-blocker.

Also, sotalol was used for the prevention of AF in cardiac surgery, showing to reduce the incidence of postoperative $\mathrm{AF}[14,20]$ compared to placebo or to other $\beta$-blocker such as atenolol [37], metoprolol [38] or propranolol [39] but it had no impact on the length of hospital stay, risk of strokes or mortality.

Because of its important side effects (bradycardia and torsade de pointes), the use of sotalol in the treatment of POAF is limited, especially in patients with electrolyte disturbances. This is why sotalol has class IIb indication, according to ESC guidelines for AF prevention in cardiac surgery patients [9].

The use of amiodarone is also beneficial for the postoperative prevention of atrial arrhythmias, reducing the length of hospitalization, decreasing the incidence of postoperative AF, stroke, and postoperative ventricular tachyarrhythmia, being without effect on postoperative mortality [40].

The use of $\beta$-blockers or other antiarrhythmic drugs is limited in some patient's subgroups with severe LV dysfunction and active bronchospasm or having cardiac conduction abnormalities. In this case, ivabradine, a specific inhibitor of the $I_{\mathrm{f}}$ current in the sinoatrial node can be an optimal alternative. Ivabradine is a pure heartrate lowering agent in patients with sinus rhythm, the main advantage being that ivabradine does not affect myocardial contractility, blood pressure, intracardiac conduction, or ventricular repolarisation.

In the BEAUTIFUL study (patients with coronary artery disease and LV systolic dysfunction-left ventricular ejection fraction of less than 40\%), ivabradine reduced the incidence of endpoints related to coronary artery disease (admission to hospital for fatal and non-fatal acute myocardial infarction) [24]. Ivabradine, also can be used in conjunction with $\beta$-blockers in patients with coronary artery disease and impaired LV systolic function, this combination showing an improvement on coronary artery disease outcomes in patients with heart rates of $70 \mathrm{bpm}$ or more [24]. These results suggest that further lowering of heart rate has beneficial effects on coronary disease outcomes [24].

In the SHIFT study, performed in patients with stable symptomatic chronic HF and a LV ejection fraction of $35 \%$ or lower, with a resting heart rate of $70 \mathrm{bpm}$ or higher, ivabradine added to optimal standard treatment significantly reduced major risks associated with HF: cardiovascular death or hospital admission for worsening HF [23].

The results of these two studies supporting the importance of heart rate reduction with ivabradine for improvement of clinical outcomes in HF or coronary artery disease with systolic LV dysfunction were the rationale for using ivabradine alone or in combination with metoprolol for prevention of postoperative rhythm disorders and reduction of subsequent morbidity, mortality and associated economic costs in patients with conduction abnormalities or LV systolic dysfunction undergoing open heart surgery $[20,21]$.

Ivabradine is a new and safe alternative to decrease heart rate in postoperative cardiac surgery patients, without affecting blood pressure; it allows us to reduce the heart rate in a group of patients in whom side effects of "target doses" of $\beta$-blockers are a major concern. To decrease the oxygen consumption and to reduce heart rate is the target in the immediate postoperative period and that cannot be obtained in all patients by increasing the $\beta$ - 
blockers. That is the role of ivabradine's use in different indication in the postoperative period of cardiac surgery patients who developed tachyarrhithmias or AF which could not be controlled by $\beta$-blocker due to conduction abnormalities, LV systolic dysfunction or hypotension with $\beta$-blocker therapy.

In our case, it was more effective the use of combined therapy (ivabradine and metoprolol) for the prevention of POAF and other tachyarrhythmias than monotherapy with metoprolol or ivabradine alone in the postoperative period for the patients with CABG or valvular replacement.

The patients treated with ivabradine improved the QOL, shortened the hospitalization stay, and shortened the time of immobilization in the postoperative period. It also decreased the incidence of supraventricular or ventricular arrhythmias.

Because of the association of $\mathrm{AF}$ and other postoperative tachiarrhythmias with increased morbidity and mortality and longer, more expensive hospital stays, we also defined a composite efficacy and safety endpoint of 30-day mortality, in-hospital AF/arrhythmias, in-hospital AV block/need for pacing, or inhospital HF worsening. Ivabradine as monotherapy or in combination with metoprolol was superior to metoprolol in respect to the composite efficacy and safety endpoints for prevention of rhythm control and prevention of rhythm disorders after cardiac surgery.

\section{Study Limitations}

The absence of a washout period- $\beta$-blocker therapy was not stopped before the randomization, $85 \%$ of our patients having preoperative $\beta$-blocker therapy. The practice in our department was to routinely continue preoperative $\beta$-blocker therapy without any pause and changing the active principle according to the study group.

About $30 \%$ of the patients with previous episodes of AF received prior to the inclusion in the study an antiarrhythmic agent such as amiodarone or sotalol.

Because $85 \%$ of our patients were treated with $\beta$-blockers before entering the study, it is possible that the results to be influenced, meaning that the effect of ivabradine be superior to metoprolol in the first 2 days at baseline (the time of removal of metoprolol is 15-35 h). The lack of a washout period for patients on beta-blocker at time of study entry may have a minor influence results in terms of overrating of ivabradine in the first 2 days of the study, while it lasts metoprolol elimination.

Given these limitations, the study can be considered exploratory and hypothesis generating.

\section{CONCLUSION}

The QOL (measured by the EuroQol questionnaire) was improved in patients treated with ivabradine combined with metoprolol due to shortened immobilization during the immediate postoperative period $(2.0 \pm 3.0$ days in the Metoprolol group, $1.1 \pm 3.0$ days in Metoprolol + Ivabradine group), shorter hospital stay (in the Metoprolol group was $10.2 \pm 6.3$ days, compared to $8.5 \pm 6.8$ days in Metoprolol + Ivabradine group), less atrial or ventricular arrhythmias (cardiac rhythm was continuously monitored in the ICU. During further hospital stay, subsequent ECG tests and a $24 \mathrm{~h}$ ECG Holter monitoring was carried out prior to discharge), less worsening HF, less need for 
permanent pacing, as well as due to lack of significant side effects.

The heart rate reduction in the early postoperative period after cardiac surgery in patients with conduction abnormalities or LV dysfunction with combined ivabradine and metoprolol therapy emerged as the best treatment in this trial, considering the ivabradine efficacy and safety profile.

As a final conclusion, ivabradine is an attractive alternative pharmacological strategy for rhythm and heart rate control in the early postoperative period in patients undergoing open heart surgery (CABG and/or valvular replacement) with relative or absolute contraindications to $\beta$-blocker therapy.

\section{ACKNOWLEDGMENTS}

Dr. L. Iliuta is the guarantor for this article, and takes responsibility for the integrity of the work as a whole. No funding or sponsorship was received for this study or publication of this article.

Conflict of interest. Dr L. Iliuta and Dr. M Rac-Albu declare no conflict of interest.

Compliance with ethics guidelines. The study protocol was approved by the institute Management and Ethics Committee. All procedures followed were in accordance with the ethical standards of the responsible committee on human experimentation (institutional and national) and with the Helsinki Declaration of 1975, as revised in 2000 and 2008. Informed consent was obtained from all patients for being included in the study.

Open Access. This article is distributed under the terms of the Creative Commons
Attribution Noncommercial License which permits any noncommercial use, distribution, and reproduction in any medium, provided the original author(s) and the source are credited.

\section{REFERENCES}

1. Mina K, Chung MD. Cardiac surgery: postoperative arrhythmias. Crit Care Med. 2000;28(10 Suppl): N136-44.

2. Helgadottir S, Sigurdsson MI, Ingvarsdottir IL, Arnar DO, Gudbjartsson T. Atrial fibrillation following cardiac surgery: risk analysis and longterm survival. J Cardiothorac Surg. 2012;7:87.

3. Taylor G, Malik S, Colliver J, et al. Usefulness of AF as a predictor of stroke after isolated coronary artery bypass grafting. Am J Cardiol. 1987;60:905-7.

4. Zaman AG, Archbold RA, Helft G, Paul EA, Curzen NP, Mills PG. AF after coronary artery bypass surgery: a model for preoperative risk stratification. Circulation. 2000;101:1403-8.

5. Auer J, Weber T, Berent R, et al. Risk factors of postoperative atrial fibrillation after cardiac surgery. J Card Surg. 2005;20(5):425-31.

6. Mathew JP, Fontes ML, Tudor IC, et al. AF after coronary artery surgery - a multicenter risk index for AF after cardiac Surgery. Am Heart J. 2004;147:636-43.

7. Stebbins D, Igidbashian L, Goldman SM, et al. Clinical outcome of patients who develop AF after coronary artery bypass graft surgery. PACE. 1995;18:798.

8. Mathew JP, Parks R, Savino JS, et al. AF following coronary artery bypass graft surgery: predictors, outcomes, and resource utilization. JAMA. 1996;276:300-6.

9. Camm AJ, Kirchhof P, Lip GYH, et al. Guidelines for the management of AF. Eur Heart J. 2010;31: 2369-429.

10. Kaireviciute D, Blann AD, Balakrishnan B, et al. Characterisation and validity of inflammatory biomarkers in the prediction of post-operative $\mathrm{AF}$ in coronary artery disease patients. Thromb Haemost. 2010;104:122-7.

11. Kalman JM, Munawar M, Howes LG, et al. AF after coronary artery bypass grafting is associated with sympathetic activation. Ann Thorac Surg. 1995;60:1709-15. 
12. Kaireviciute D, Aidietis A, Lip GY. AF following cardiac surgery: clinical features and preventative strategies. Eur Heart J. 2009;30:410-25.

13. Mathew JP, Fontes ML, Tudor IC, et al. A multicenter risk index for AF after cardiac surgery. JAMA. 2004;291:1720-9.

14. Aranski SF, ShawDP, Adams DH, Rizzo RJ, Couper GS, VanderVliet M. Predictors of AF after coronary artery surgery. Current trends and impact on hospital resources. Circulation 1996;94:390-7.

15. Ascione R, Caputo M, Calori G, Lloyd CT, Underwood MJ, Angelini GD. Predictors of AF after conventional and beating heart coronary surgery: a prospective, randomized study. Circulation. 2000;102:1530-5.

16. Iliuta L, Christodorescu R, Filipescu D, et al. Prevention of perioperative AF with betablockers in coronary surgery: betaxolol versus metoprolol. Interact Cardiov Thorac Surg. 2010;9:89-93.

17. Burgess DC, Kilborn MJ, Keech AC. Interventions for prevention of postoperative $\mathrm{AF}$ and its complications after cardiac surgery: a metaanalysis. Eur Heart J. 2006;27:2846-57.

18. Lucio Ede A, Flores A, Blacher C, et al. Effectiveness of metoprolol in preventing $\mathrm{AF}$ and flutter in the postoperative period of coronary artery bypass graft surgery. Arquivos Brasileiros de Cardiologia. 2004;82:42-46; 37-41.

19. Matangi MF, Neutze JM, Graham KJ, et al. Arrhythmia prophylaxis after aorto-coronary bypass: the effect of minidose propanolol. J Thorac Cardiov Sur. 1985;89:439-43.

20. Crystal E, Garfinkle MS, Connolly SS, et al.: Interventions for preventing post-operative $\mathrm{AF}$ in patients undergoing heart surgery. Cochrane Database Syst Rev. 2004(4), Art. No.CD003611.

21. Crystal E, Thorpe KE, Connolly SJ, et al. Metoprolol prophylaxis against postoperative AF increases length of hospital stay in patients not on preoperative $\beta$-blockers: the beta blocker length of stay (BLOS) trial. Heart. 2004;90:941-2.

22. Eagle KA, Guyton RA, Davidoff R, et al. ACC/AHA 2004 guideline update for coronary artery bypass graft surgery: a report of the American College of Cardiology/American Heart Association Task Force on Practice Guidelines (Committee to Update the 1999 Guidelines for Coronary Artery Bypass Graft Surgery). Circulation. 2004;110:340-437.

23. Swedberg K, Komajda M, Böhm M, et al. SHIFT Investigators: Ivabradine and outcomes in chronic heart failure (SHIFT): a randomised placebocontrolled study. Lancet. 2010;9744:875-85.

24. Fox K, Ford I, Steg PG, BEAUTIFUL Investigators, et al. Ivabradine for patients with stable coronary artery disease and left-ventricular systolic dysfunction (BEAUTIFUL): a randomised, doubleblind, placebo-controlled trial. Lancet. 2008;9641: 807-16.

25. Iliuta L, Enache R. Ivabradine versus beta-blockers in patients with conduction abnormalities or left ventricular dysfunction undergoing coronary artery bypass grafting. InTech Publishing. 2011, p. $355-368$.

26. Yue-Chun L, Teng Z, Na-Dan Z, Li-Sha G, Qin L, Xue-Qiang G, Jia-Feng L. Comparison of effects of ivabradine versus carvedilol in murine model with the Coxsackievirus B3-induced viral myocarditis. PLoS ONE. 2012;7:e39394.

27. Rabin R, de Charro F. EQ-5D: a measure of health status from the EuroQol Group. Ann Med. 2001;33:337-43.

28. Villareal RP, Hariharan R, Liu B, et al. Postoperative $\mathrm{AF}$ and mortality after coronary artery bypass surgery. J Am Coll Cardiol. 2004;43:742-8.

29. Tamis JE, Steinberg JS. AF independently prolongs hospital stay after coronary artery bypass surgery. Clin Cardiol. 2000;23:155-9.

30. Reed G III, Singer DE, Picard EH, DeSanctis RW. Stroke following coronary-artery bypass surgery. A case-control estimate of the risk from carotid bruits. New Engl J Med. 1988;319:1246-50.

31. Martinez EA, Bass EB, Zimetbaum P. Pharmacologic control of rhythm: American College of Chest Physicians guidelines for the prevention and management of postoperative AF after cardiac surgery. Chest. 2005;128(2 Suppl):48S-55S.

32. Lamb RK, Prabhakar G, Thorpe JA, et al. The use of atenolol in the prevention of supraventricular arrhythmias following coronary artery surgery. Eur Heart J. 1988;9:32-36.

33. Kamei M, Morita S, Hayashi Y, et al. Carvedilol versus metoprolol for the prevention of AF after offpump coronary bypass surgery: rationale and design of the carvedilol or metoprolol postrevascularization AF controlled trial (COMPACT). Cardiovasc Drug Ther. 2006;20:219-27.

34. Celik T, Iyisoy A, Jata B, et al. Betablockers for the prevention of AF after coronary artery bypass surgery: carvedilol versus metoprolol. Int J Cardiol. 2009;135:393-6. 
35. Daudon P, Corcos T, Gandjbakhch I, et al. Prevention of AF or flutter by acebutolol after coronary bypass grafting. Am J Cardiol. 1986;58:933-66.

36. White HD, Antman EM, Glynn MA, et al. Efficacy and safety of timolol for prevention of supraventricular tachyarrhythmias after coronary artery bypass surgery. Circulation. 1984;70:479-84.

37. Sanjuan R, Blasco M, Carbonell N, et al. Preoperative use of sotalol versus atenolol for AF after cardiac surgery. Ann Thorac Surg. 2004;77:838-43.

38. Parikka H, Toivonen L, Heikkila $\mathrm{L}$, et al. Comparison of sotalol and metoprolol in the prevention of AF after coronary artery bypass surgery. J Cardiovasc Pharm. 1998;31:67-73.

39. Suttorp MJ, Kingma JH, Tjon Joe Gin RM, et al. Efficacy and safety of low- and high-dose sotalol versus propranolol in the prevention of supraventricular tachyarrhythmias early after coronary artery bypass operations. J Thorac Cardiov Surg. 1990;100:921-26.

40. Bagshaw SM, Galbraith PD, Mitchell LB, et al. Prophylactic amiodarone for prevention of $\mathrm{AF}$ after cardiac surgery: a meta-analysis. Ann Thorac Surg. 2006;82:1927-37. 